\title{
Externalizing behavior problems and influencing factors among Hui nationality left-behind children in rural areas of China
}

\section{Xue Yu(D yuxue200704@126.com )}

Beijing First Hospital of Integrated Traditional Chinese and Western Medicine- First Affiliated Hospital of Institute of Basic Theory, China Academy of Chinese Medical Sciences https://orcid.org/0000-0002-15564904

\section{Lingling Wang}

Luoyang Fifth People Hospital

Miaomiao Liu

Yinchuan University of Energy

\section{Qiuli Li}

Ningxia Medical University

\section{Xiuying Dai}

Ningxia Medical University

\section{Primary research}

Keywords: Hui nationality, left-behind children, externalizing behavior problems, rural areas

Posted Date: March 19th, 2021

DOI: https://doi.org/10.21203/rs.3.rs-19515/v3

License: (1) This work is licensed under a Creative Commons Attribution 4.0 International License. Read Full License 


\section{Abstract}

Background: Externalizing behavior problems (EBP) is being increasingly viewed as a public health problem. Previous study showed that the detection rate of behavioral problems of Hui nationality left-behind children (LBC) was far higher than that of Han nationality LBC. However, existing literature has not reported EBP of Hui nationality LBC. The purpose of current study was to investigate the prevalence EBP and its influencing factors among Hui nationality LBC in rural areas of China.

Methods: A cross-sectional study was conducted among school students from the southern rural areas in Ningxia, China (2012-2013). The general self-made questionnaire, Egma Minnen av Bardndosnauppforstran (EMBU), Eysenck Personality Questionnaire (EPQ for Children), Piers-Harri Children's Self-concept Scale (PHCSS) and Achenbach's Child behavior Checklist (CBCL for parents) were used to investigate the related information. Descriptive analysis and logistic regressions were conducted.

Results: The prevalence of EBP in Hui nationality LBC and non-left-behind children(non-LBC) were $12.01 \%$ $(46 / 383)$ and $8.57 \%(49 / 572)$, respectively, and there was no significant difference between them $\left(\chi^{2}=3.04\right.$, $P=0.08)$. Multivariate logistic regression analysis showed that low self-concept of behavioral $(O R=13.71)$, introversion ( $O R=11.19)$ and intermediate personality $(O R=9.35)$ were risk factors for EBP of Hui nationality LBC, while the intermediate type of mother refusal and denial $(O R=0.43)$ was their protective factor.

Conclusions: Our findings suggest that parental migration is a risk factor for EBP among Hui nationality LBC in rural China. Influencing factors should be considered when formulating relevant policies and undertaking intervention measures in the future, so as to reduce EBP of Hui nationality LBC.

\section{Background}

Children's behavioral problems refer to the abnormal behaviors that affect children's social functions, including behavioral and emotional problems. An important distinction in the field of child psychology is the separation between 'externalizing' and 'internalizing' disorders[1]. Externalizing behavior problems (EBP) refer to a group of behavior problems that are evident in children's outward behavior[2]. This study focuses on childhood's externalizing behavior, which is a major risk factor for later juvenile delinquency, adult crime, and violence[3].

Since the reform and opening up, with the continuous development of industrialization and urbanization in China during the past several decades, a large number of surplus rural labor force has transferred to the city for employment, forming a group of special population of left-behind children(LBC). The term "LBC" refer to children less than 16 years old staying in their rural hometowns and villages for more than half a year because both or one of their parents migrate to an urban area for work ,and were taken care of by their grandparents, relatives, neighbors, single parent, siblings or themselves[4]. According to the research report released by china women's federation[5] in 2013, there were about 61.02 million LBC in China, who were concentrated in the underdeveloped rural areas in the central and western regions, accounting for 37.7 percent of the total number of rural children and 21.9 percent of the total number of children in China. LBC are in a critical period of psychological growth, and the long-term absence of the role of parents will cause lasting negative impacts on their psychological development [6, 7], which is prone to cognitive deviation and higher behavioral problems[810], among which delinquency, aggression and other externalizing behavior problems(EBP) are being 
increasingly viewed as a public health problem. If these problems are not corrected, it will seriously affect the healthy development of adolescents and lead to social problems such as juvenile delinquency and adult violence[11]. Moreover, previous research also reported that the EBP could cause mental illness. For example, Mathysseke at al.[12]found that the social problems, thought problems and externalizing scales predicted adolescent panic attack onset. Roza[13] found that the EBP in children and adolescents were predictive of anxiety disorders in young adulthood.

Ningxia hui autonomous region is located in the northwest of China, with a population of 6.3014 million. According to the unified calculation of the national bureau of statistics, the initial calculation of Ningxia's GDP in 2019 is 374.848 billion yuan, ranking 29th among 31 provinces in China and relatively backward in economy. Main ethnic population are Hui and Han in Ningxia, among which Hui population accounts for about $1 / 3$ of the total population of the autonomous region, accounting for $98.24 \%$ of the minority population of the autonomous region. The hui population in the southern mountainous region of Ningxia accounted for more than 60 percent. Xiji and haiyuan, two national-level poverty-stricken counties in the southern mountainous region, are the gathering places of Hui nationality, with severe water shortage, barren land, closed transportation, economy backwardness and large labor export. However, few studies to date on Hui nationality LBC were reported in China. Zhao MM et al.[14]showed that the mental health of LBC in rural areas was worse than that of non-LBC in Guyuan of China. Feng YT et al.[15]took yongning county as the investigation site and found that the detection rate of behavioral problems of Hui nationality LBC was $31.25 \%$, which was far higher than $17.84 \%$ of Han nationality LBC. Previous studies have shown that children's psychological behavioral problems are related to a variety of factors, including children's personality characteristics[16], selfconcept[17-19], parenting behaviors[20, 21] , and family environment factors[19]and so on. Du YS showed that family structure and environment were closely related to children's behavior development [22], and LBC as special family structure will have more emotional[23] and social behavior problems[24]. Existing research results showed that parent migration had a considerable adverse impact on children's health outcomes [25]. A previous study showed that parental migration had a negative impact on children's EBP [26]. Some studies have shown that changes in family structure in early childhood[27], poor family communication and reduced positive reappraisal of mothers[28]are statistically associated with later increased EBP in children. What's more, another study found children exposed to maternal intimate partner violence (IPV)were more likely to have borderline to clinical level scores on EBP[29]. Similarly, Bair-Merritt MH documented that significant associations between maternal IPV and externalizing[30]. However, existing literature has not reported EBP of Hui nationality LBC, and there is still a lack of research with large sample sizes that could reveal the actual background of EBP in these population.

Therefore, this present study aims to answer three research questions. Firstly, we investigated the prevalence of EBP and special syndromes in Hui nationality LBC. Secondly, given the potential impacts of individual characteristics, self-concept and family environment on children's mental health, this study explored the relationship between multilevel risk factors and children's EBP. Finally, this study explored the EBP of Hui LBC under the influence of Hui culture background. Through the investigation of 383 Hui LBC, this study explored the related factors of EBP in Hui LBC, which is of great significance promoting their mental health, formulating relevant ethnic policies, and maintaining social stability. These findings also provided important implications for future research and intervention measures. 


\section{Methods}

\section{Subjects}

Data were obtained from a cross-sectional survey conducted in two counties Xiji, Haiyuan of Ningxia hui autonomous region in southern rural mountains from December 2012 to September 2013. Using multistage stratified random cluster sampling methods, we selected six primary schools, 5 junior high schools in XinYing Township, HongYao township, XingLong town, Xi 'An town, ShuTai township form Xiji and Haiyuan countries. One class from grade 1 to grade 9 was randomly selected from each school. A total of 41 classes were selected for the survey. A total of 2000 questionnaires were distributed, and 1905 valid ones were recovered, with an effective rate of $95.25 \%$, among which 955 (50.13\%) Hui nationality children were the subjects of this study. In these 955 participants, 383 were LBC while 755 were not. Inclusion criteria for LBC: who stay in rural areas for more than half a year while both parents or single parents working outside. They are taken care of by their grandparents, relatives, neighbors, single parent, or themselves. They are aged from 6 to 16 years old. Exclusion criteria: whose both parents or single parent has worked outside for less than 6 months; Having a serious physical or mental illness.

\section{Measuring tool}

Sociodemographic characteristics: Participants completed a questionnaire which included their gender, age, nationality, academic performance, caregiver and their education level, parents' education level and occupation, frequency contact with parents, frequency contact with parents, etc.

Achenbach's Child behavior Checklist (CBCL): Achenbach's Child behavior Checklist was developed by American psychologist Dr. Achenbach. It was published in 1976[1], and revised for the first time in 1983 [31]. The scale is suitable for children aged 4 to 16 and has been widely used all over the world. In this study, the school-age version of checklist included 113 items to identify children's behavior problems. Parents or caregivers who were familiar with the children used the 3-point Likert scale $(0=$ not true, $1=$ somewhat or sometimes true, 2 = very true or often true) to report children's behaviors in the past six months. Male and female students each have 8-9 kinds of specific symptoms, including schizoid, depressed, uncommunicative, obsessive-compulsive, somatic complaints, social withdrawal, hyperactivity, aggressive and delinquent behavior. Through principal component analysis of the above factors, we get two dimensions of internalizing behavior problem and externalizing behavior problems (EBP). China introduced and standardized this scale and formulated the national and Hunan norm[32,33]. The Cronbach's alpha is about 0.73 for externalization behavior. We calculated the raw score of specific symptoms and compared it with that of the norm. The higher the score, the more obvious the behavior problems was. In this survey, boys' EBP included hyperactivity, delinquent and aggression; Girls' EBP include hyperactivity, sexual problems, delinquent, aggression and cruel. In this study, Children whose raw scores were above the upper threshold of the norm sample in at least one syndrome were considered to have behavioral disorders[33]. The score of EBP was calculated by adding the corresponding syndrome scores, and the repeated items were not accumulated.

Egna Minnen av Barndoms Uppfostran(EMBU): This scale was developed by C. Perris et al, department of psychiatry, Umea University in Sweden in 1980 to assess parents' attitudes and behaviors. 
This study adopted the Chinese version revised by Yue DM[34]in1993. The revised version takes into account the cultural differences between Chinese and Western individuals, with a total of 66 items and 11 syndromes. Among them, there are 58 items of paternal rearing style, with a total of 6 syndromes, including emotional warmth and understanding, punishment and strictness, over-interference, favoring subjects, refusal and denial and over-protection. There were 57 items of maternal rearing style, with a total of 5 syndromes, including emotional warmth and understanding, over-interference and over-protection, refusal and denial, punishment and strictness and favoring subjects. The test-retest reliability is between 0.58 and 0.82 . The split-half reliability is between 0.50 and 0.91 , and the internal consistency reliability is between 0.59 and 0.88 . This scale was later used by many researchers and obtained good reliability and validity. The scale is suitable for anyone who is children, if it is a single parent family or is an only child, and deal with related topics to make "unsuitable" for an answer. Each item is scored according to never $=1$, occasionally $=2$, often $=3$, always $=4$, and unsuitable $=0$. The score was determined according to the norm mean score \pm standard deviation. Factor scores < mean score - standard deviation was low score, > mean score + standard deviation was high score, and the rest was medium score.

Eysenck Personality Questionnaire (EPQ) : Personality was assessed using Eysenck personality questionnaire for Chinese children which revised by YX Gong, et al[35]. This version has 88 true-false items and includes four sub-scales: evaluating neuroticism $(\mathrm{N})$, extroversion-introversion $(\mathrm{E})$, psychoticism $(\mathrm{P})$, and Lie $(\mathrm{L})$ dimensions. Part of the scale is the reverse scoring title. We calculated raw scores of each sub-scale, and converted into standard T points, standard T < 38.5 for typical low score, 38.5 to 43.3 for tended to low score, 43.3 to 56.7 for the medium type, 56.7 to 61.5 as the tendency to high score, $>61.5$ for the typical high score. The Cronbach's alpha was 0.70 for the total scale, $0.76,0.76,0.88$, and 0.77 for $E, P, N$, and $L$, respectively.

Piers Harris Child's self-concept Scale (PHCSS): Children's self-concept Scale (PHCSS) is a self-rating Scale for Children compiled by American psychologists Piers and Harris in 1969 and revised in 1974. It is mainly used to evaluate Children's self- concept. It is composed of 80 items, including 6 sub-scales of behavioral, intelligence and school status, physical appearance and attributes, anxiety, popularity, happiness and satisfaction. The answer is 1, no 0, part of the question is the reverse score. In 2002, Su LY introduced and revised this scale, and formulated Chinese city norm[34,36], with good reliability and validity. The score was determined according to the norm mean score \pm standard deviation, the scores < mean score -standard deviation was low score, the scores $>$ mean score + standard deviation was high score, and the rest was medium score. The higher the total score or the score of a factor was, the stronger the self-concept or self-concept in a certain aspect was. A high "behavior" score indicates that the child is behaving appropriately, and a high "anxiety" score indicates that the child is in good mood and not anxious. The half-reliability of PHCSS scale 0.82 and the Cronbach's alpha was 0.858 , which was suitable for the measurement of children's self-concept in China.

\section{Procedures}

We have been supported by the local education bureau and the leaders of the surveyed schools.

The head teacher issued the informed consent for the survey to the parents' Wechat group, and explained the purpose and significance of the survey in detail to the students and their parents / guardians, as well as the way of filling in the questionnaire and the confidential method.

Written informed consent signed by the participants and their parents / guardians was taken back to school. 
The head teacher then handed the participants' written informed consent to the investigators. After the questionnaire is distributed in class, the students are required to complete the questionnaire within the prescribed time (60-80 minutes), and the questionnaire is collected by on-site inspection. For students from grade one to grade three, the researchers read each item to the subjects in neutral, non suggestive language, and asked them to understand and then answer. Achenbach children's Behavior Checklist (CBCL) was taken home by the students and handed over to the guardian to fill in after the communication between the head teacher and the parents. It was collected within 3 days. For the missing students or incomplete answers, we conducted a family supplementary survey. 122 LBC of Hui nationality were investigated. All investigators were trained in advance

\section{Statistical analyses}

Epidata3.0 software was used to establish the database, double input data, SPSS19.0 for Windows software was used for statistical analysis. Chi square test was used for categorical variables and $t$-test for continuous variables. Binary logistic regression was used to analyze the risk factors of EBP in Hui nationality LBC. The value of $p$ takes the probability of two sides, and $a$ takes 0.05 as a test standard.

\section{Results}

\section{Sample Description}

As seen in Table 1, a total of 955 hui nationality children were investigated. Among them, 383 children were hui nationality LBC, $50.65 \%$ of which were male. In terms of the age distribution, hui LBC aged 6 to 11 years accounted for $29.77 \%\left(M_{\text {age }}=12.86\right.$ years, $S D=2.77$ years $)$. There were 572 hui nationality non-LBC, among which 263 were male (46.98\%). There were 181 children (31.64\%) aged $6 \sim 11$ years old $\left(\mathrm{M}_{\text {age }}=12.62\right.$ years, $\mathrm{SD}=2.49$ years). There was no significant difference in demographic characteristics between hui LBC and non-LBC (see Table 1 for details). 
Table 1

The demographic characteristics between hui nationality LBC and non-LBC.

\begin{tabular}{|c|c|c|c|c|c|c|c|}
\hline \multirow[t]{2}{*}{ Characteristics } & & \multicolumn{2}{|c|}{$\operatorname{LBC}(n=383)$} & \multicolumn{2}{|c|}{ non-LBC(n = 572) } & \multirow[t]{2}{*}{$x^{2}$} & \multirow{2}{*}{$\begin{array}{l}P \\
\text { value }\end{array}$} \\
\hline & & $\mathbf{N}$ & $\%$ & $\mathbf{N}$ & $\%$ & & \\
\hline \multirow[t]{2}{*}{ Gender } & male & 194 & 50.65 & 263 & 45.98 & \multirow[t]{2}{*}{2.01} & \multirow[t]{2}{*}{0.16} \\
\hline & female & 189 & 49.35 & 309 & 54.02 & & \\
\hline \multirow[t]{2}{*}{ Age group } & aged $6-11$ & 114 & 29.77 & 181 & 31.64 & \multirow[t]{2}{*}{0.38} & \multirow[t]{2}{*}{0.55} \\
\hline & aged 12-16 & 269 & 70.23 & 391 & 68.36 & & \\
\hline Mean age(years) & Mean (SD) & $12.86(2.77)$ & & $12.62(2.49)$ & & $1.40^{\Delta}$ & 0.16 \\
\hline \multirow[t]{2}{*}{ Only child } & yes & 15 & 3.92 & 16 & 2.80 & \multirow[t]{2}{*}{0.92} & \multirow[t]{2}{*}{0.34} \\
\hline & no & 368 & 96.08 & 556 & 97.20 & & \\
\hline \multirow[t]{2}{*}{ Mother alive } & yes & 375 & 97.91 & 567 & 99.13 & \multirow[t]{2}{*}{2.52} & \multirow[t]{2}{*}{0.11} \\
\hline & no & 8 & 2.09 & 5 & 0.87 & & \\
\hline \multirow[t]{2}{*}{$\begin{array}{l}\text { Father's education } \\
\text { level }\end{array}$} & $\begin{array}{l}\text { junior high } \\
\text { school or } \\
\text { higher }\end{array}$ & 77 & 20.10 & 123 & 21.50 & \multirow[t]{2}{*}{0.27} & \multirow[t]{2}{*}{0.60} \\
\hline & $\begin{array}{l}\text { primary school } \\
\text { or lower }\end{array}$ & 306 & 79.90 & 449 & 78.50 & & \\
\hline \multirow[t]{2}{*}{$\begin{array}{l}\text { Maternal education } \\
\text { level }\end{array}$} & $\begin{array}{l}\text { junior high } \\
\text { school or } \\
\text { higher }\end{array}$ & 29 & 7.57 & 49 & 8.57 & \multirow[t]{2}{*}{0.30} & \multirow[t]{2}{*}{0.58} \\
\hline & $\begin{array}{l}\text { primary school } \\
\text { or lower }\end{array}$ & 354 & 92.43 & 523 & 91.43 & & \\
\hline \multirow[t]{2}{*}{$\begin{array}{l}\text { Caregiver's education } \\
\text { level }\end{array}$} & $\begin{array}{l}\text { junior high } \\
\text { school or } \\
\text { higher }\end{array}$ & 76 & 19.84 & 123 & 21.50 & \multirow[t]{2}{*}{0.38} & \multirow[t]{2}{*}{0.54} \\
\hline & $\begin{array}{l}\text { primary school } \\
\text { or lower }\end{array}$ & 307 & 80.16 & 449 & 78.50 & & \\
\hline \multirow{3}{*}{$\begin{array}{l}\text { Academic } \\
\text { performance }\end{array}$} & good & 53 & 13.84 & 102 & 17.83 & \multirow[t]{3}{*}{3.86} & \multirow[t]{3}{*}{0.15} \\
\hline & moderate & 262 & 68.41 & 358 & 62.59 & & \\
\hline & poor & 68 & 17.75 & 112 & 19.58 & & \\
\hline \multirow[t]{2}{*}{$\begin{array}{l}\text { Frequency of contact } \\
\text { with teachers }\end{array}$} & $\begin{array}{l}\text { at least once a } \\
\text { week }\end{array}$ & 26 & 6.79 & 38 & 6.64 & \multirow[t]{2}{*}{5.52} & \multirow[t]{2}{*}{0.14} \\
\hline & $\begin{array}{l}\text { at least once a } \\
\text { month }\end{array}$ & 47 & 12.27 & 92 & 16.08 & & \\
\hline
\end{tabular}




\begin{tabular}{|c|c|c|c|c|c|c|c|}
\hline \multicolumn{2}{|l|}{ Characteristics } & \multicolumn{2}{|c|}{ LBC(n = 383) } & \multicolumn{2}{|c|}{ non-LBC(n = 572) } & \multirow[t]{2}{*}{$x^{2}$} & \multirow{2}{*}{$\begin{array}{l}P \\
\text { value }\end{array}$} \\
\hline & & $\mathbf{N}$ & $\%$ & $\mathbf{N}$ & $\%$ & & \\
\hline & $\begin{array}{l}>\text { once a } \\
\text { month }\end{array}$ & 222 & 57.96 & 291 & 50.87 & & \\
\hline & never contact & 88 & 22.98 & 151 & 26.40 & & \\
\hline \multirow[t]{2}{*}{ Father alive } & yes & 373 & 97.39 & 561 & 98.08 & \multirow[t]{2}{*}{0.51} & \multirow[t]{2}{*}{0.48} \\
\hline & no & 10 & 2.61 & 11 & 1.92 & & \\
\hline \multirow[t]{2}{*}{ Father's occupation } & farmers & 299 & 60.84 & 470 & 76.92 & \multirow[t]{2}{*}{2.46} & \multirow[t]{2}{*}{0.12} \\
\hline & non-farmers & 84 & 39.16 & 102 & 23.18 & & \\
\hline \multirow[t]{2}{*}{ Mother's occupation } & farmers & 299 & 74.93 & 474 & 82.87 & \multirow[t]{2}{*}{3.43} & \multirow[t]{2}{*}{0.06} \\
\hline & Not farmers & 84 & 25.07 & 98 & 17.13 & & \\
\hline \multirow[t]{2}{*}{ Parents' divorced } & yes & 16 & 4.18 & 20 & 3.50 & \multirow[t]{2}{*}{0.35} & \multirow[t]{2}{*}{0.56} \\
\hline & no & 367 & 95.82 & 552 & 96.50 & & \\
\hline
\end{tabular}

\section{The prevalence of EBP in hui nationality children}

Table 2 showed that the overall prevalence of EBP of 955 hui nationality children was $9.95 \%$ (95/955), among whom 383 hui LBC had a prevalence of $12.01 \%$ (46/383) and 572 hui non-LBC was $8.57 \%(49 / 572)$. There was no significant statistical difference between the two group $\left(\chi^{2}=3.04, P=0.08\right)$. And there was no significant statistical difference in the prevalence of EBP among hui nationality LBC and non-LBC in gender.

Table 2

Prevalence of EBP among different categories of hui nationality children (\%)

\begin{tabular}{|c|c|c|c|c|c|c|}
\hline \multicolumn{2}{|c|}{ Variables } & \multicolumn{5}{|c|}{ externalizing behavior problems } \\
\hline & & Sample size & Positive number & Prevalence rate & $\chi^{2}$ & $P$-value \\
\hline \multirow[t]{3}{*}{ LBC } & yes & 383 & 46 & 12.01 & \multirow[t]{2}{*}{3.04} & \multirow[t]{2}{*}{0.08} \\
\hline & non & 572 & 49 & 8.57 & & \\
\hline & total & 955 & 95 & 9.95 & - & \\
\hline \multirow[t]{2}{*}{ Boys } & LBC & 194 & 22 & 11.34 & \multirow[t]{2}{*}{2.83} & \multirow[t]{2}{*}{0.09} \\
\hline & non-LBC & 263 & 18 & 6.84 & & \\
\hline \multirow[t]{2}{*}{ Girls } & LBC & 189 & 24 & 12.70 & \multirow[t]{2}{*}{0.85} & \multirow[t]{2}{*}{0.36} \\
\hline & non-LBC & 309 & 31 & 10.03 & & \\
\hline
\end{tabular}




\section{Prevalence of specific syndromes and EBP in hui nationality children aged 6-11}

When examining the prevalence of specific syndromes and EBP in hui nationality children, we found that there was no significant difference in the prevalence of specific syndromes and EBP between hui nationality LBC and non-LBC aged $6 \sim 11$

Table 3: Prevalence specific syndromes and EBP in hui nationality children aged 6-11 (\%)

\begin{tabular}{|c|c|c|c|c|c|c|c|c|}
\hline & \multicolumn{4}{|c|}{ Boys } & \multicolumn{4}{|c|}{ Girls } \\
\hline & $\mathrm{LBC} \square \mathrm{n}=56 \square$ & $\begin{array}{l}\text { non-LBC } \\
\square n=88 \square\end{array}$ & $\chi^{2}$ & $P$-value & $\begin{array}{l}\mathrm{LBC} \\
\square \mathrm{n}=58 \square\end{array}$ & $\begin{array}{l}\text { non-LBC } \\
\square n=93 \square\end{array}$ & $\chi^{2}$ & $P$-value \\
\hline & 5๑8.93॰ & 7๑7.95ロ & 0.04 & 0.84 & - & - & - & - \\
\hline & $8 \square 14.29 \square$ & 7๑7.95॰ & 1.47 & 0.23 & 2๑3.45॰ & 6ロ6.45॰ & 0.18 & 0.67 \\
\hline ive & 4ロ7.14ロ & 10ロ11.36॰ & 0.70 & 0.41 & - & - & - & - \\
\hline pulsive & $4(7.14)$ & $9(10.23)$ & 0.40 & 0.53 & - & - & - & - \\
\hline aints & $1(1.79)$ & $7(7.95)$ & 1.45 & 0.23 & 5ロ8.62凸 & 11⒒83ロ & 0.39 & 0.53 \\
\hline wal & 2๑3.57๑ & 5ロ5.68\ & 0.03 & 0.86 & $2(3.45)$ & 7 (7.53) & 0.46 & 0.50 \\
\hline & $0 \square 0 \square$ & $0 \square 0 \square$ & - & - & $2 \square 3.45 \square$ & 5๑5.38๑ & 0.02 & 0.88 \\
\hline & 2๑3.57๑ & $1 \square 1.14 \square$ & 0.16 & 0.69 & 1๑1.72॰ & 3ロ3.23॰ & 0.00 & 0.97 \\
\hline & 3๑5.36๘ & $4 \square 4.55 \square$ & 0.00 & 1.00 & $3(5.17)$ & $5(5.38)$ & 0.00 & 1.00 \\
\hline & - & - & - & - & $2(3.45)$ & $6(6.45)$ & 0.18 & 0.67 \\
\hline & - & - & - & - & $6 \square 10.34 \square$ & 6ロ6.45ロ & 0.30 & 0.58 \\
\hline sive & - & - & - & - & 7 (12.07) & 9₫9.68) & 0.22 & 0.64 \\
\hline iehavior & 4๑7.14\ & 5๑5.68\ & 0.00 & 1.00 & 8ロ13.79ロ & 14ロ15.05ロ & 0.05 & 0.83 \\
\hline
\end{tabular}

Prevalence of specific syndromes and EBP in hui nationality children aged 12-16 Table 4 showed that there were significant statistical differences in physical complaints, uncommunicative, obsessive-compulsive, hostility and delinquent (all $P<0.05$ ) between hui nationality LBC and non-LBC in boys aged $12-16$. And there was no significant difference in the prevalence of specific syndromes between hui nationality LBC and nonLBC in girls aged 12-16 (all $P=>0.05$ ). 
Table 4

Prevalence specific syndromes and EBP in hui nationality children aged $12-16$ years (\%)

\begin{tabular}{|c|c|c|c|c|c|c|c|c|}
\hline \multirow[t]{2}{*}{ syndromes } & \multicolumn{4}{|l|}{ Boys } & \multicolumn{4}{|l|}{ Girls } \\
\hline & $\begin{array}{l}\text { LBC } \\
(n=138)\end{array}$ & $\begin{array}{l}\text { non-LBC } \\
(n= \\
175)\end{array}$ & $x^{2}$ & $\begin{array}{l}P \\
\text { value }\end{array}$ & $\begin{array}{l}\text { LBC } \\
(n=131)\end{array}$ & $\begin{array}{l}\text { non-LBC } \\
(n=216)\end{array}$ & $x^{2}$ & $\begin{array}{l}P \\
\text { value }\end{array}$ \\
\hline $\begin{array}{l}\text { Somatic } \\
\text { Complaints }\end{array}$ & 19(13.77) & $10(5.71)$ & 5.95 & 0.02 & $7(5.34)$ & $6(2.78)$ & 0.86 & 0.35 \\
\hline Schizoid & $13(9.42)$ & $7(4.00)$ & 3.79 & 0.05 & $24(18.32)$ & $34(15.74)$ & 0.39 & 0.53 \\
\hline uncommunicative & 14(10.14) & $6(3.43)$ & 5.82 & 0.02 & - & - & - & - \\
\hline Immature & $6(4.35)$ & $4(2.29)$ & 1.06 & 0.30 & 10(7.63) & $7(3.24)$ & 3.38 & 0.07 \\
\hline $\begin{array}{l}\text { Obsessive- } \\
\text { Compulsive }\end{array}$ & $20(14.49)$ & $12(6.86)$ & 4.90 & 0.03 & - & - & - & - \\
\hline Hostile & 16(11.59) & $5(2.86)$ & 9.41 & 0.00 & - & - & - & - \\
\hline Delinquent & $13(9.42)$ & $3(1.71)$ & 9.45 & 0.00 & $8(6.11)$ & $7(3.24)$ & 1.62 & 0.20 \\
\hline Aggressive & $8(5.80)$ & $7(4.00)$ & 0.55 & 0.46 & $8(6.11)$ & $5(2.31)$ & 2.29 & 0.13 \\
\hline Hyperactive & $9(6.52)$ & $5(2.86)$ & 2.43 & 0.12 & - & - & - & - \\
\hline $\begin{array}{l}\text { Anxious- } \\
\text { Obsessive }\end{array}$ & - & - & - & - & $7(5.34)$ & $9(4.17)$ & 0.26 & 0.61 \\
\hline $\begin{array}{l}\text { Depressed- } \\
\text { Withdrawal }\end{array}$ & - & - & - & - & $10(7.63)$ & 18(8.33) & 0.05 & 0.82 \\
\hline Cruel & - & - & - & - & 12(9.16) & 11(5.09) & 2.18 & 0.14 \\
\hline $\begin{array}{l}\text { Externalizing } \\
\text { behavior }\end{array}$ & 18(13.04) & 13(7.43) & 2.73 & 0.10 & $16(12.21)$ & 17(7.87) & 1.79 & 0.18 \\
\hline
\end{tabular}

\section{The scores of CBCL syndromes between hui nationality LBC and non-LBC aged 6-11}

As shown in the Table 5, except that the hyperactivity factor score of hui boys LBC was significantly lower than that of hui boys non-LBC $(t=2.71, \mathrm{P}<0.05)$, the difference of other syndromes was not statistically significant $(P>0.05)$. 
Table 5

CBCL syndromes scores between hui nationality LBC and non-LBC aged 6-11 years (Mean \pm SD)

\begin{tabular}{|c|c|c|c|c|c|c|c|c|}
\hline \multirow[t]{2}{*}{ syndromes } & \multicolumn{4}{|l|}{ Boys } & \multicolumn{4}{|l|}{ Girls } \\
\hline & $\begin{array}{l}\mathrm{LBC}(\mathrm{n}= \\
56)\end{array}$ & $\begin{array}{l}\text { non-LBC(n } \\
=88)\end{array}$ & $t$ & $P$ & $\begin{array}{l}\operatorname{LBC}(n \\
=58)\end{array}$ & $\begin{array}{l}\text { non-LBC }(n \\
=93)\end{array}$ & $\begin{array}{l}t \\
\text { value }\end{array}$ & $\begin{array}{l}P \text { - } \\
\text { value }\end{array}$ \\
\hline Schizoid & $\begin{array}{l}2.13 \pm \\
2.45\end{array}$ & $2.67 \pm 2.53$ & -1.28 & 0.20 & - & - & - & - \\
\hline Depressed & $\begin{array}{l}3.11 \pm \\
4.79\end{array}$ & $3.35 \pm 4.43$ & -0.31 & 0.75 & $\begin{array}{l}4.72 \pm \\
4.44\end{array}$ & $4.91 \pm 5.38$ & -0.23 & 0.82 \\
\hline uncommunicative & $\begin{array}{l}2.11 \pm \\
2.52\end{array}$ & $2.39 \pm 3.00$ & -0.58 & 0.56 & - & - & - & - \\
\hline $\begin{array}{l}\text { Obsessive- } \\
\text { Compulsive }\end{array}$ & $\begin{array}{l}2.80 \pm \\
3.71\end{array}$ & $3.58 \pm 4.11$ & -1.15 & 0.25 & - & - & - & - \\
\hline $\begin{array}{l}\text { Somatic } \\
\text { Complaints }\end{array}$ & $\begin{array}{l}1.34 \pm \\
2.12\end{array}$ & $1.93 \pm 2.92$ & -1.32 & 0.19 & $\begin{array}{l}3.24 \pm \\
3.75\end{array}$ & $3.55 \pm 4.16$ & -0.46 & 0.65 \\
\hline $\begin{array}{l}\text { Social- } \\
\text { Withdrawal }\end{array}$ & $\begin{array}{l}1.34 \pm \\
2.08\end{array}$ & $2.01 \pm 2.40$ & -1.72 & 0.09 & $\begin{array}{l}3.03 \pm \\
3.05\end{array}$ & $3.08 \pm 3.71$ & -0.07 & 0.94 \\
\hline Hyperactive & $\begin{array}{l}2.20 \pm \\
2.25\end{array}$ & $3.39 \pm 3.01$ & -2.71 & 0.01 & $\begin{array}{l}3.05 \pm \\
3.19\end{array}$ & $3.53 \pm 3.74$ & -0.80 & 0.42 \\
\hline Aggressive & $\begin{array}{l}4.98 \pm \\
6.19\end{array}$ & $5.45 \pm 5.86$ & -0.03 & 1.00 & $\begin{array}{l}4.62 \pm \\
4.37\end{array}$ & $4.80 \pm 5.80$ & -0.20 & 0.84 \\
\hline Delinquent & $\begin{array}{l}1.52 \pm \\
2.69\end{array}$ & $2.16 \pm 2.55$ & -1.44 & 0.38 & $\begin{array}{l}0.74 \pm \\
1.60\end{array}$ & $0.61 \pm 1.24$ & 0.55 & 0.58 \\
\hline $\begin{array}{l}\text { Schizoid- } \\
\text { Obsessive }\end{array}$ & - & - & - & - & $\begin{array}{l}1.74 \pm \\
2.69\end{array}$ & $1.68 \pm 2.90$ & 0.14 & 0.89 \\
\hline Sex problems & - & - & - & - & $\begin{array}{l}0.93 \pm \\
1.55\end{array}$ & $1.23 \pm 1.76$ & -1.05 & 0.30 \\
\hline Cruel & - & - & - & - & $\begin{array}{l}0.98 \pm \\
1.90\end{array}$ & $1.06 \pm 1.78$ & -0.27 & 0.79 \\
\hline $\begin{array}{l}\text { Externalizing } \\
\text { behavior }\end{array}$ & $\begin{array}{l}8.07 \pm \\
9.19\end{array}$ & $9.48 \pm 8.75$ & -0.92 & 0.36 & $\begin{array}{l}9.33 \pm \\
9.20\end{array}$ & $\begin{array}{l}10.23 \pm \\
11.09\end{array}$ & -0.52 & 0.61 \\
\hline
\end{tabular}

\section{The scores of $C B C L$ syndromes between hui nationality LBC and non-LBC aged 12-16}

As shown in the Table 6, the hui nationality boys LBC had higher scores in schizoid, somatic complaints, uncommunicative, obsessive-compulsive, hostile, delinquent, aggression, hyperactivity and EBP than that of boys non-LBC $(P<0.05)$. The hui nationality girls LBC got higher scores in aggressive, cruel and EBP than that of girls non-LBC $(P<0.05)$. 
Table 6

CBCL syndromes scores between hui nationality LBC and non-LBC aged $12-16$ years (Mean \pm SD)

\begin{tabular}{|c|c|c|c|c|c|c|c|c|}
\hline \multirow[t]{2}{*}{ syndromes } & \multicolumn{4}{|l|}{ Boys } & \multicolumn{4}{|l|}{ Girls } \\
\hline & $\begin{array}{l}\text { LBC } \\
(n=138)\end{array}$ & $\begin{array}{l}\text { non-LBC }(n \\
=175)\end{array}$ & $t$ & $\begin{array}{l}P \text { - } \\
\text { value }\end{array}$ & $\begin{array}{l}\text { LBC } \\
(n=131)\end{array}$ & $\begin{array}{l}\text { non-LBC ( } \\
=216)\end{array}$ & $t$ & $\begin{array}{l}P- \\
\text { value }\end{array}$ \\
\hline Schizoid & $\begin{array}{l}3.66 \pm \\
3.18\end{array}$ & $2.74 \pm 2.58$ & 2.75 & 0.01 & $\begin{array}{l}2.69 \pm \\
2.83\end{array}$ & $2.39 \pm 2.56$ & 1.02 & 0.31 \\
\hline $\begin{array}{l}\text { Somatic } \\
\text { Complaints }\end{array}$ & $\begin{array}{l}5.16 \pm \\
5.30\end{array}$ & $3.65 \pm 4.19$ & 2.73 & 0.01 & $\begin{array}{l}2.70 \pm \\
3.04\end{array}$ & $2.43 \pm 2.78$ & 0.87 & 0.39 \\
\hline Uncommunicative & $\begin{array}{l}6.44 \pm \\
6.04\end{array}$ & $4.22 \pm 4.67$ & 3.57 & 0.00 & - & - & - & - \\
\hline Immature & $\begin{array}{l}1.72 \pm \\
2.10\end{array}$ & $1.36 \pm 1.84$ & 1.64 & 0.10 & $\begin{array}{l}5.11 \pm \\
4.38\end{array}$ & $4.25 \pm 3.86$ & 1.91 & 0.06 \\
\hline $\begin{array}{l}\text { Obsessive- } \\
\text { Compulsive }\end{array}$ & $\begin{array}{l}3.16 \pm \\
3.17\end{array}$ & $2.28 \pm 2.45$ & 2.69 & 0.01 & - & - & - & - \\
\hline Hostile & $\begin{array}{l}4.62 \pm \\
4.81\end{array}$ & $3.33 \pm 3.33$ & 2.68 & 0.01 & - & - & - & - \\
\hline Delinquent & $\begin{array}{l}3.08 \pm \\
3.78\end{array}$ & $2.29 \pm 2.71$ & 2.08 & 0.04 & $\begin{array}{l}4.63 \pm \\
4.32\end{array}$ & $3.76 \pm 3.56$ & 1.92 & 0.06 \\
\hline Aggression & $\begin{array}{l}7.04 \pm \\
6.47\end{array}$ & $5.62 \pm 5.61$ & 2.09 & 0.04 & $\begin{array}{l}6.71 \pm \\
6.13\end{array}$ & $5.37 \pm 5.09$ & 2.11 & 0.04 \\
\hline Hyperactive & $\begin{array}{l}3.96 \pm \\
3.49\end{array}$ & $2.98 \pm 3.04$ & 2.64 & 0.01 & - & - & - & - \\
\hline $\begin{array}{l}\text { Anxious- } \\
\text { Obsessive }\end{array}$ & - & - & - & - & $\begin{array}{l}7.27 \pm \\
6.13\end{array}$ & $6.40 \pm 6.02$ & 1.31 & 0.19 \\
\hline $\begin{array}{l}\text { Depressed- } \\
\text { Withdrawal }\end{array}$ & - & - & - & - & $\begin{array}{l}5.73 \pm \\
4.95\end{array}$ & $5.19 \pm 4.73$ & 1.00 & 0.32 \\
\hline Cruel & - & - & - & - & $\begin{array}{l}2.19 \pm \\
3.49\end{array}$ & $1.36 \pm 1.96$ & 2.50 & 0.01 \\
\hline $\begin{array}{l}\text { Externalizing } \\
\text { behavior }\end{array}$ & $\begin{array}{l}12.04 \pm \\
10.96\end{array}$ & $9.41 \pm 8.94$ & 2.28 & 0.02 & $\begin{array}{l}12.31 \pm \\
10.98\end{array}$ & $9.78 \pm 8.45$ & 2.26 & 0.03 \\
\hline
\end{tabular}

\section{Univariate analysis results}

First, chi-square test was conducted on the counting data that affected the incidence of EBP of hui nationality LBC, it was found that the frequency of contact with teachers, academic performance and parents' working out conditions were related to EBP of hui nationality LBC (all $P<0.05$ ) (see Table 7 for details). Second, $t$ - test was conducted on the measurement data, the results showed that behavior, anxiety, popularity, happiness and satisfaction, total score of self-concept, father punishment and strictness, father favoring subjects, father refusal and denial, father over-protection, mother over-interference and over-protection, mother refusal and 
denial, mother punishment and strictness, Extroversion-introversion (E), Psychoticism (P),Neuroticism(N), and Lie (L)were related to EBP of hui nationality LBC(see Table 8 for details).. 
Table 7

Univariate analysis of the influence of general demographic data on EBP of hui nationality LBC $(n=383)$

\begin{tabular}{|c|c|c|c|c|c|}
\hline \multirow[t]{2}{*}{ Characteristics } & & \multicolumn{4}{|c|}{ externalizing behavior problems } \\
\hline & & $\begin{array}{l}\text { total } \\
\text { number }\end{array}$ & $\begin{array}{l}\text { positive } \\
\text { number }\end{array}$ & $x^{2}$ & $\begin{array}{l}P \text { - } \\
\text { value }\end{array}$ \\
\hline \multirow[t]{2}{*}{ Gender } & male & 194 & 22 & \multirow[t]{2}{*}{0.17} & \multirow[t]{2}{*}{0.68} \\
\hline & female & 189 & 24 & & \\
\hline \multirow[t]{2}{*}{ Caregiver's education level } & $\begin{array}{l}\text { junior high school or } \\
\text { higher }\end{array}$ & 76 & 10 & \multirow[t]{2}{*}{0.12} & \multirow[t]{2}{*}{0.73} \\
\hline & primary school or lower & 307 & 36 & & \\
\hline \multirow[t]{2}{*}{ Age group } & aged $6-11$ & 114 & 12 & \multirow[t]{2}{*}{0.34} & \multirow[t]{2}{*}{0.56} \\
\hline & aged $12-16$ & 269 & 34 & & \\
\hline \multirow[t]{2}{*}{ Father's education level } & $\begin{array}{l}\text { junior high school or } \\
\text { higher }\end{array}$ & 77 & 5 & \multirow[t]{2}{*}{2.78} & \multirow[t]{2}{*}{0.10} \\
\hline & primary school or lower & 306 & 41 & & \\
\hline \multirow[t]{2}{*}{ Maternal education level } & $\begin{array}{l}\text { junior high school or } \\
\text { higher }\end{array}$ & 29 & 5 & \multirow[t]{2}{*}{0.37} & \multirow[t]{2}{*}{0.55} \\
\hline & primary school or lower & 354 & 41 & & \\
\hline \multirow{4}{*}{$\begin{array}{l}\text { Frequency of contact with } \\
\text { teachers }\end{array}$} & at least once a week & 26 & 2 & \multirow[t]{4}{*}{10.01} & \multirow[t]{4}{*}{0.02} \\
\hline & at least once a month & 47 & 4 & & \\
\hline & > once a month & 222 & 21 & & \\
\hline & never contact & 88 & 19 & & \\
\hline \multirow[t]{3}{*}{ Academic performance } & good & 53 & 2 & \multirow[t]{3}{*}{14.98} & \multirow[t]{3}{*}{0.00} \\
\hline & moderate & 262 & 27 & & \\
\hline & poor & 68 & 17 & & \\
\hline \multirow[t]{2}{*}{ Only child } & yes & 15 & 3 & \multirow[t]{2}{*}{0.32} & \multirow[t]{2}{*}{0.57} \\
\hline & no & 368 & 43 & & \\
\hline \multirow[t]{2}{*}{ Parents' divorced } & yes & 16 & 1 & \multirow[t]{2}{*}{0.11} & \multirow[t]{2}{*}{0.74} \\
\hline & no & 367 & 45 & & \\
\hline \multirow[t]{2}{*}{ Working out conditions } & Single parents go out & 252 & 24 & \multirow[t]{2}{*}{4.31} & \multirow[t]{2}{*}{0.04} \\
\hline & Both parents go out & 131 & 22 & & \\
\hline \multirow[t]{2}{*}{ Father's occupation } & farmers & 233 & 28 & 0.00 & 1.00 \\
\hline & Not farmers & 150 & 18 & & \\
\hline
\end{tabular}




\begin{tabular}{|llllll|} 
Mother's occupation & farmers & 287 & 30 & 2.63 & 0.11 \\
\cline { 2 - 5 } & Not farmers & 96 & 16 & & \\
Father alive & yes & 373 & 46 & 0.43 & 0.22 \\
Mother alive & no & 10 & 0 & & \\
& yes & 375 & 45 & 0.00 & 1.00 \\
\hline & no & 8 & 1 & & \\
\hline
\end{tabular}

Table 8: Univariate analysis of the influence of measurement data on EBP of hui nationality LBC(n=383) $($ Mean \pm SD)

\begin{tabular}{|c|c|c|c|c|}
\hline \multirow[t]{2}{*}{ ariables } & \multicolumn{2}{|c|}{ externalizing behavior problems } & \multirow[t]{2}{*}{$t$} & \multirow[t]{2}{*}{$P$-value } \\
\hline & no $1337 \square$ & yes $\square 46 \square$ & & \\
\hline \multicolumn{5}{|l|}{$\overline{\text { ICSS }}$} \\
\hline Behavior & $11.77 \pm 2.54$ & $9.20 \pm 2.72$ & 6.41 & 0.00 \\
\hline Intellectual and School Status & $8.34 \pm 3.28$ & $7.46 \pm 3.16$ & 1.73 & 0.09 \\
\hline Physical Appearance and Attributes & $5.60 \pm 2.76$ & $5.52 \pm 2.69$ & 0.18 & 0.86 \\
\hline Anxiety & $8.21 \pm 2.52$ & $6.93 \pm 2.59$ & 3.21 & 0.00 \\
\hline Popularity & $8.17 \pm 2.06$ & $6.87 \pm 2.26$ & 3.96 & 0.00 \\
\hline Happiness and Satisfaction & $6.58 \pm 1.95$ & $5.91 \pm 2.19$ & 2.15 & 0.03 \\
\hline Total Score of self-concept & $49.37 \pm 10.73$ & $40.59 \pm 10.72$ & 4.03 & 0.00 \\
\hline \multicolumn{5}{|l|}{ MBU } \\
\hline Emotional warmth and understanding $(\mathrm{F})$ & $44.31 \pm 9.59$ & $43.04 \pm 10.25$ & 0.84 & 0.41 \\
\hline Punishment and strictness(F) & $19.28 \pm 5.81$ & $23.67 \pm 8.12$ & -3.54 & 0.00 \\
\hline Over-interference(F) & $18.64 \pm 4.61$ & $20.41 \pm 6.23$ & -1.86 & 0.07 \\
\hline Favoring subjects(F) & $8.71 \pm 3.10$ & $10.00 \pm 3.67$ & -2.59 & 0.01 \\
\hline Refusal and denial(F) & $9.16 \pm 3.12$ & $11.20 \pm 4.29$ & -3.11 & 0.00 \\
\hline Over-protection(F) & $10.28 \pm 2.58$ & $11.20 \pm 2.78$ & -2.23 & 0.03 \\
\hline Emotional warmth and understanding(M) & $47.46 \pm 9.35$ & $44.72 \pm 8.80$ & 1.88 & 0.06 \\
\hline Over- interference and over-protection(M) & $34.07 \pm 6.62$ & $36.48 \pm 6.89$ & -2.30 & 0.02 \\
\hline Refusal and denial(M) & $13.01 \pm 4.03$ & $16.13 \pm 4.82$ & -4.19 & 0.00 \\
\hline Punishment and strictness(M) & $14.15 \pm 4.50$ & $17.61 \pm 5.53$ & -4.06 & 0.00 \\
\hline Favoring subjects(M) & $9.91 \pm 2.98$ & $10.48 \pm 3.40$ & -1.20 & 0.23 \\
\hline \multicolumn{5}{|l|}{ PQ } \\
\hline Extroversion-introversion (E) & $15.10 \pm 4.19$ & $13.30 \pm 3.29$ & 2.80 & 0.01 \\
\hline Psychoticism (P) & $10.29 \pm 4.52$ & $11.80 \pm 4.13$ & -2.15 & 0.03 \\
\hline Neuroticism (N) & $4.53 \pm 2.76$ & $6.43 \pm 2.96$ & -4.36 & 0.00 \\
\hline Lie (L) & $13.44 \pm 4.25$ & $11.59 \pm 3.72$ & 2.82 & 0.01 \\
\hline
\end{tabular}

Note: father(F), mother(M)

\section{Multivariate non-conditional logistic regression analysis results}

The total score of EBP of hui nationality LBC was taken as the dependent variable (negative $=0, p o s i t i v e=1$ ), and 19 factors with statistical significance in univariate analysis, including behavior, anxiety, popularity, happiness and satisfaction, total score of self-concept, father punishment and strictness, father favoring subjects, father refusal and denial, father overprotection, mother over-interference and overprotection, mother refusal and denial, mother punishment and strictness, Extroversion-

introversion,Psychoticism,Neuroticism,Lie,frequency contact with teacher, academic performance and parents working out conditions were taken as the independent variables. Multivariate non-conditional logistic regression analysis, by using forward conditions, into the standard of alpha $=0.05$, exclusion criteria $=0.10$, showed that low self-awareness of behavior, introversion and intermediate personality were the risk factors for the EBP of hui nationality LBC, while the intermediate type of mother refusal and denial was the protective factor for the EBP of hui nationality LBC. As shown in the Table 9. 
Table 9

Results of multivariate logistic regression analysis predicting EBP of hui nationality LBC $(\mathrm{n}=383)$

\begin{tabular}{|c|c|c|c|c|c|c|c|}
\hline variables & reference & & B & S.E. & Wald & $\begin{array}{l}P \\
\text { value }\end{array}$ & $\begin{array}{l}\text { OR } \\
(95 \% \mathrm{Cl})\end{array}$ \\
\hline \multirow[t]{2}{*}{ behavior } & High & Low & 2.50 & 1.06 & 5.580 & 0.02 & $\begin{array}{l}12.23(1.53 \\
\sim 97.60)\end{array}$ \\
\hline & & Medium & 0.88 & 1.06 & 0.693 & 0.41 & $\begin{array}{l}2.42(0.30 \\
\sim 19.33)\end{array}$ \\
\hline \multirow[t]{2}{*}{$\begin{array}{l}\text { mother refusal and } \\
\text { denial }\end{array}$} & High & Low & -1.90 & 1.07 & 3.153 & 0.08 & $\begin{array}{l}0.15(0.02 \\
\sim 1.22)\end{array}$ \\
\hline & & Medium & -0.97 & 0.37 & 6.771 & 0.01 & $\begin{array}{l}0.38(0.18 \\
\sim 0.79)\end{array}$ \\
\hline \multirow[t]{2}{*}{$\begin{array}{l}\text { extroversion- } \\
\text { introversion }\end{array}$} & High & Low & 2.49 & 1.06 & 5.496 & 0.02 & $\begin{array}{l}12.10(1.51 \\
\sim 97.33)\end{array}$ \\
\hline & & Medium & 2.27 & 1.05 & 4.688 & 0.03 & $\begin{array}{l}9.70(1.24 \\
\sim 75.86)\end{array}$ \\
\hline \multirow[t]{3}{*}{$\begin{array}{l}\text { frequency of contact } \\
\text { with teachers }\end{array}$} & $\begin{array}{l}\text { at least once } \\
\text { a week }\end{array}$ & $\begin{array}{l}\text { at least once } \\
\text { a month }\end{array}$ & -0.01 & 0.95 & 0.000 & 1.00 & $\begin{array}{l}1.00(0.16 \\
\sim 6.40)\end{array}$ \\
\hline & & $\begin{array}{l}\text { > once a } \\
\text { month }\end{array}$ & 0.69 & 0.81 & 0.724 & 0.40 & $\begin{array}{l}1.98(0.41 \\
\sim 9.60)\end{array}$ \\
\hline & & never contact & 1.440 & 0.82 & 3.090 & 0.08 & $\begin{array}{l}4.22(0.85 \\
\sim 21.00)\end{array}$ \\
\hline constant & & & -5.83 & 1.60 & 13.327 & 0.00 & 0.00 \\
\hline
\end{tabular}

\section{Discussion}

This study results showed that 95 out of 955 hui children had externalizing behavior problems, with a prevalence of $9.95 \%$. Among the 383 hui LBC, 46 persons had EBP, with a prevalence of $12.01 \% .49$ out of 572 hui nationality non-LBC had EBP, with a prevalence of $8.57 \%$. The prevalence of EBP was not statistically significant difference between hui nationality LBC and non-LBC. However, this study[37] found that the overall prevalence of behavioral problems of hui nationality LBC (25.1\%) was significantly higher than that of hui nationality non-LBC (20.5\%).The results was lower than the prevalence $41.3 \%$ reported by Wei-min Xu[8], But higher than $12.97 \%$ reported from China's 22 provinces to investigate children's behavior problems [38], and $17.6 \%$ of Xu Jing reported [39], also higher than $10.49 \%$ of shui LBC's behavior problem prevalence reported by Guo GW[40] .In terms of the prevalence of EBP, the prevalence of hui nationality LBC was $12.01 \%$, which was significantly higher than $4.03 \%$ of school-age children in rural areas of Harbin reported by Wu LJ et al[41]. We analyzed that it could be related to ethnic cultural differences, different scales of use and differences in social development, but it was close to $13.2 \%$ of the prevalence of EBP in LBC reported by $\mathrm{Hu} \mathrm{H}$ et al[42]. This study also found that there was no significant difference in the prevalence of EBP between boys and girls of hui LBC, which was consistent with the existing research results[41]. 
In this study, it was found that among hui nationality LBC aged 6-11 years old, the top four prevalence of CBCL specific syndromes of male subjects were: depression(14.3\%),schizoid(8.9\%),obsessivecompulsive(7.1\%), uncommunicative(7.1\%)and EBP(7.1\%). The top four prevalence of CBCL specific syndromes of female subjects were EBP (13.8\%), schizoid-obsessive (12.1\%), cruelty (10.3\%) and somatic complaints (8.6\%). There was no significant difference in prevalence of specific syndromes and EBP between hui nationality LBC and non-LBC aged $6 \sim 11$, which was not consistent with that reported by liu SM et al[43].

Among hui nationality LBC aged 12-16 years old, the top four prevalence of CBCL specific syndromes of male students was: obsessive-compulsive (14.5\%), somatic complaints (13.8\%), EBP (13.0\%) and hostility (11.6\%). The top four prevalence of CBCL specific syndromes for female was schizoid (18.3\%), EBP (12.6\%), cruelty (9.2\%), depression (7.6\%) and immaturity (7.6\%). This study found that with the growth of age, the prevalence of EBP of hui nationality male LBC showed an increasing trend, while the prevalence of hui nationality female LBC showed no increasing trend, but it was found that hui nationality female LBC aged 12-16 had significantly higher EBP than female non-LBC. This study suggest that children aged 12-16 with EBP not only may negatively affect their outside world, but also may be psychologically suffering internally. In other words, hui left-behind children aged 12-16 have the phenomenon of co-morbidity between externalizing and internalizing behavior problems[44], especially boys.

This study found that the score of hyperactivity factor of hui nationality LBC aged 6-11 years old was significantly lower than that of hui non-LBC, and there were no significant statistically difference in other factors of CBCL and EBP of hui nationality LBC compared with non-LBC. This was worthy of our attention, our study indicated that hui nationality LBC in low age groups might lead to their behavior convergence due to far away from parents and their inferiority complex. Another reason is that children at this age are in primary school. Because they are far away from their parents, they learn and master certain interpersonal skills. Compared with the psychological rebellion of middle school students when they entered adolescence, primary school students had simple and peaceful psychology and relatively few behavioral problems.

Our study showed that hui nationality male LBC aged 12-16 years old had higher scores on schizoid, somatic complaints, uncommunicative, obsessive-compulsive, hostility, delinquent, aggression, hyperactivity and externalizing behavior problems than those of male non-LBC, indicating that older hui male LBC were more likely to have behavioral problems, which was consistent with that reported by Wei-min Xu[8]. The reason may be related to boy's different physiological characteristics, personality traits and family education environment. Our study also found that female hui nationality LBC aged 12-16 years old got higher scores in aggression, cruelty and EBP than those of non-LBC, suggesting that the behavior problems of the older hui nationality female LBC were mainly characterized by aggression and cruelty, and indicating that hui girls LBC were prone to externalizing behavior problems due to lack of family affection after entering adolescence. Thus, hui nationality LBC aged 12-16 are faced with a wider range of problems, including internalizing problems such as somatic complaints, depression and schizoid, as well as externalizing behavior problems such as delinquent, hyperactivity and aggression. After entering junior high school, because of parents and teachers pay too much attention to children's academic performance, children's learning pressure becomes larger, which may have an impact on their psychology. 
In addition, due to parents' migrant work, lack of parental care and discipline, the older they get, the greater their psychological needs will be.

This was consistent with the increasing trend of emotional and behavioral problems in the process of children entering adolescence[45], suggesting that we should focus on hui nationality senior LBC, which was inconsistent with existing studies[41], and it was analyzed that our research objects were different from theirs.

The above results revealed that there were more behavioral problems among hui nationality LBC aged $12 \sim 16$ years old, which were worthy of our attention.

The results of univariate analysis showed that children's gender, caregiver's education level, parents' education level, parents divorced and only child had no effect on EBP of hui nationality LBC, which was in line with the previous research results[46],but was inconsistent with the existing study reported that parents divorced may exacerbate the negative effects of parental migration[47]. However, the frequency of contact with teachers, academic performance, and parents' working out situation seemed to have an influence, but they were not independent influences in multivariate analysis, which was quite inconsistent with the existing studies at home and abroad[48-51]. The possible reason is that the subjects of this study are Hui nationality left behind children in poor mountainous areas who influenced by the religious culture of Hui nationality, the culture of Hui nationality may have a certain regulatory effect on internalizing behavior problems, or it may be related to the small sample size of this study. These results suggest that they may play an indirect role in children's personality development and self-concept development.

The results of multivariate analysis showed that low behavior score, introversion and intermediate personality were the risk factors for EBP of hui nationality LBC, while intermediate type of mother refusal and denial was the protective factor for EBP of hui nationality LBC. This result indicated that the low score of self-concept behavior tended to cause hui nationality LBC to have higher EBP, which was consistent with the existing research[52] that pointed out a significant negative correlation between behavioral problems and self-concept. Our research also revealed that negative parenting styles, such as too much or too little maternal refusal and denial, will increase the EBP of hui nationality LBC, which was consistent with the positive correlation between EBP and authoritarian parenting style reported by Zhu WF et al[53]. Family is not only an important cultural carrier, but also is the first learning setting children are exposed to. Parental rearing style is considered a specific educational medium through which Chinese culture and social values are passed on to children. As a result of the impact that traditional Chinese culture and the education system have on parents, a hierarchy still exists between parents and children in most Chinese families.

On the one hand, If a child's behavior goes against the will of the caregiver, rejection or punishment may occur. Previous studies[54,55] suggested that corporal punishment by parents was strongly associated with later children externalizing behavior, such as aggression, criminality and antisocial behavior. On the other hand, hui nationality culture is a kind of national culture formed on basis of Hui culture and Chinese traditional culture. Mosque, market and Hui community are interrelated and interdependent, and constitute three important links of Hui life: worship, commerce and residence[56]. In families with plenty of time and a strong religious atmosphere, most hui children can enrich their understanding of the religion they believe in, either through the conscious guidance of elders' words and deeds or through the religious activities they are exposed to. Hui people believe in Islam and are deeply influenced by Islamic culture. They emphasize obedience in the ethical 
norms of their daily life. This culture has exerted a subtle influence on Hui children through family and social education, making them unconsciously compromise instead of insisting on their own opinions when confronted with conflicts between their own and others'. With the deepening of China's reform and opening up, the construction of market economy and the influx of a large number of labor force into cities, this pattern has been damaged[57], so that these left-behind children can't inherit the characteristics of the hui culture, become more aggressive and indiscipline. Our study suggested that only moderate denial by mothers might have a protective effect on the development of EBP in hui nationality LBC, and that parent-based intervention had been proved to be effective in improving children's EBP[58]. Moreover, children learn moral values and social conventions through a process of socialization, much of which involves parenting styles[59], and it has been proved that moral education can promote the moral development of adolescents and hopefully reduce their EBP[60].

Our research had several limitations. Firstly, due to the limited conditions, the sample population was single, involving only five township schools in two project demonstration counties, which limited the scalability of the research results and affected the external validity of this study. Secondly, we collected information from caregivers or parents about children's behavioral problems, which might lead to bias. Thirdly, the scores of the nationwide norm sample used in this study were collected through an epidemiology survey in 1992. It might lead to limitations when we used it to assess children's behavioral problems in current studies, as China has undergone dramatic development in the past decades. Finally, this survey is a cross-sectional study, and it is impossible to obtain detailed information about the complete development process and the overall trends of EBP over time in hui nationality LBC. Furthermore, the CBCL is adopted as a screening scale in this survey. Therefore, the prevalence of EBP and CBCL syndromes of hui nationality LBC cannot be used as the basis for the diagnosis of children's emotional and behavioral problems. In order to provide more compelling evidence concerning influencing factors on EBP of hui nationality LBC, a longitudinal and prospective study is recommended to explore the mechanism of how these risk factors lead to EBP at individual and group levels.

\section{Conclusion}

This study showed a higher prevalence of EBP as well as specific syndromes of behavioral problems in hui nationality LBC. Our findings suggested that parental migration was a potential risk factor for EBP among hui nationality LBC in rural China. Introverted personality, intermediate personality, low self-conscious of behavior, and intermediate type of mother refusal are independent influencing factors for the occurrence of EBP of hui nationality LBC. Influencing factors should be considered when formulating relevant policies and undertaking intervention measures in the future, so as to reduce EBP of Hui nationality LBC.

\section{Abbreviations}

LBC: Left-behind children; non-LBC: non-left-behind children; EBP: externalizing behavior problems; CBCL: Achenbach's Child behavior Checklist; EMBU: Egma Minnen av Bardndosnauppforstran; EPQ: Eysenck Personality Questionnaire; PHCSS: Piers-Harri Children's Self-concept Scale.

\section{Declarations}




\section{Competing interests}

The authors declare that they have no competing interests.

\section{Consent for publication}

Not applicable.

\section{Ethics approval and consent to participate}

Ethics approval was obtained from the Ethics Committees at Ningxia Medical University. Written informed consent was obtained from the students or caregiver in advance.

\section{Funding}

This research was financially supported by grants from "twelfth five-year" National science and technology for rural areas support program (2012BAJ18B07-2). The funder had no role in study design, data analysis and interpretation, or preparation of the manuscript.

\section{Author Contributions}

Conceptualization: Yu xue, Li Qiuli.

\section{Acknowledgments}

The authors thank all participants in this study, as well as all the interviewers for data collection in this study.

\section{References}

1. Achenbach TM. The Child Behavior Profile: I. Boys aged 6-11. J Consult Clin Psychol. 1978;46(3):47888.

2. Eisenberg N, Cumberland A, Spinrad TL, Fabes RA, Shepard SA, Reiser M, et al. The relations of regulation and emotionality to children's externalizing and internalizing problem behavior. Child development. 2001;72(4):1112-34.

3. Betz LC, Childhood Violence. A Nursing Concern. Issues in Comprehensive Pediatric Nursing. 1995;18(3):149-61.

4. Duan CR, Zhou FL. Studies on Children Left Behind. population research. 2005.29 (1).

5. -36. (In Chinese).

6. China women's federation. A research report on the status of rural left-behind children in China. Chin Women's Movement. 2013. (6): 30-34. (In Chinese).

7. Zheng TG, Wang SM, Zhou SH,Shu DLi J, Zheng NX,et al.Analysis of intervertion effect on left-children students from different guardian. Chin J School Health,2010,31(12):1459-1460. (In Chinese). 
8. Zhao C, Wang F, Li L, Zhou X, Hesketh T. Long-term impacts of parental migration on Chinese children's psychosocial well-being: mitigating and exacerbating factors. Soc Psychiatry Psychiatr Epidemiol. 2017;52(6):669-77. doi:10.1007/s00127-017-1386-9. PubMed PMID: 28439622; PubMed Central PMCID: PMCPMC5487538. Epub 2017/04/26.

9. Xu WM, Tang JL, Wu D, Xu XY,Yang L. Research on present situnation of bahavior disorders of left-behind children in the countryside of Anhui province. J Appl Clin Pediatr.2007. 22(11): 852-853. (In Chinese).

10. Haight W, Black J, Sheridan K. A mental health intervention for rural, foster children from methamphetamine-involved families: Experimental assessment with qualitative elaboration. Child Youth Serv Rev. 2010;32(10):1446-57.

11. Chen SH, Liao ZG, Wang SH, Xi BR,Liu H, He SZ. Analysis of factors related to behavior problems of leftbehind children in jiangxi province. Chin J Sch Health. 2014;35(1):95-7. (In Chinese).

12. Wichers M, Gardner C, Maes HH, Lichtenstein P, Larsson H, Kendler KS. Genetic innovation and stability in externalizing problem behavior across development: a multi-informant twin study. Behavior genetics. 2013;43(3):191-201. Epub 2013/02/05. doi: 10.1007/s10519-013-9586-x. PubMed PMID: 23377846.

13. Mathyssek CM, Olino TM, Verhulst FC, van Oort FVA, van Os J. Childhood Internalizing and Externalizing Problems Predict the Onset of Clinical Panic Attacks over Adolescence: The TRAILS Study. PLoS ONE. 2012;7(12). doi:10.1371/journal.pone.0051564.

14. Roza SJ, Hofstra MB, van der Ende J, Verhulst FC. Stable prediction of mood and anxiety disorders based on behavioral and emotional problems in childhood: a 14-year follow-up during childhood, adolescence, and young adulthood. Am J Psychiatry. 2003;160(12):2116-21. doi:10.1176/appi.ajp.160.12.2116. PubMed PMID: 14638580. Epub 2003/11/26.

15. Zhao MM, Li HLi JLi LG, Wang CL,Stephen, Nicholas,Meng. QY.Studying on the influence of migrant parents on mental health of their left-behind children in rural China. Chin Health Service Management. 2012;29(1):60-3. (In Chinese).

16. Feng YT, Hu. CZ,Li QL,Liu MM,Yu X,Dai XY.Detectable rate and correlative factors.

17. of behavior problems. among Hui nationality left-behind children in rural district of Ningxia. Chin J Behavioral Medicine Brain Science. 2013;22(2):157-9. (In Chinese).

18. Van Heel M, Bijttebier P, Colpin H, Goossens L, Van Den Noortgate W, Verschueren K, et al. Investigating the interplay between adolescent personality, parental control, and externalizing problem behavior across adolescence. J Res Pers. 2019;81:176-86.

19. Wang X, Ling L, Su H, Cheng J, Jin L, Sun YH. Self-concept of left-behind children in China: a systematic review of the literature. Child Care Health Dev. 2015;41(3):346-55. doi:10.1111/cch.12172. PubMed PMID: 25039693. Epub 2014/07/22.

20. Ybrandt $\mathrm{H}$. The relation between self-concept and social functioning in adolescence. J Adolesc. 2008;31(1):1-16. doi:10.1016/j.adolescence.2007.03.004. PubMed PMID: 17467050. Epub 2007/05/01.

21. Shen JQ. Wu HJ,Chen HW.Research on correlations between aggressive behavior and rearing pa ttern, family environment and self-concept. Chin J Behavioral Medicine Science. 2006;15(8):744-5. (In Chinese).

22. Burlaka V. Externalizing behaviors of Ukrainian children: The role of parenting. Child abuse \& neglect. 2016;54:23-32. Epub 2016/02/26. doi: 10.1016/j.chiabu.2015.12.013. PubMed PMID: 26907365; 
PubMed Central PMCID: PMCPMC4834242.

23. Park S, Dotterer AM. Longitudinal associations of family stressors, fathers' warmth, and Korean children's externalizing behaviors. J Fam Psychol. 2018;32(8):1036.

24. Du YS. Tang HQ,Bao YJ,Wang YW,Zheng WZ.Behavior Problems of Children in Special Families. Chin Ment Health J. 2002;16(1):41-3. (In Chinese).

25. Wang Y-Y, Xiao L, Rao W-W, Chai J-X, Zhang S-F, Ng CH, et al. The prevalence of depressive symptoms in 'left-behind children'in China: a meta-analysis of comparative studies and epidemiological surveys. J Affect Disord. 2019;244:209-16.

26. Tang W, Wang G, Hu T, Dai Q, Xu J, Yang Y, et al. Mental health and psychosocial problems among Chinese left-behind children: A cross-sectional comparative study. J Affect Disord. 2018;241:133-41. doi: 10.1016/j.jad.2018.08.017. PubMed PMID: 30121025.

27. Meng X, Yamauchi C. Children of migrants: The cumulative impact of parental migration on children's education and health outcomes in China. Demography. 2017;54(5):1677-714.

28. Zhao J, Li Q, Wang L, Lin L, Zhang W. Latent profile analysis of left-behind adolescents' psychosocial adaptation in rural china. J Youth Adolesc. 2019;48(6):1146-60.

29. Womack SR, Taraban L, Shaw DS, Wilson MN, Dishion TJ. Family Turbulence and Child Internalizing and Externalizing Behaviors: Moderation of Effects by Race. Child Dev. 2019;90(6):e729-e44. doi:10.1111/cdev.13103. PubMed PMID: 29921025; PubMed Central PMCID: PMCPMC6301136. Epub 2018/06/20.

30. Burlaka V, Wu Q, Wu S, Churakova I. Internalizing and Externalizing Behaviors among Ukrainian Children: The Role of Family Communication and Maternal Coping. J Child Fam stud. 2019;28(5):1283-93. doi:10.1007/s10826-019-01377-w. PubMed PMID: 31031556; PubMed Central PMCID: PMCPMC6483107. Epub 2019/04/30.

31. Kernic MA, Wolf ME, Holt VL, McKnight B, Huebner CE, Rivara FP. Behavioral problems among children whose mothers are abused by an intimate partner. Child Abuse Negl. 2003;27(11):1231-46. doi:10.1016/j.chiabu.2002.12.001. PubMed PMID: 14637299.

32. Bair-Merritt MH, Ghazarian SR, Burrell L, Crowne SS, McFarlane E, Duggan AK. Understanding How Intimate Partner Violence Impacts School Age Children's Internalizing and Externalizing Problem Behaviors: A Secondary Analysis of Hawaii Healthy Start Program Evaluation Data. J Child Adolesc Trauma. 2015;8(4):245-51. Epub 2016/02/19. doi:10.1007/s40653-015-0066-8. PubMed PMID: 26889302; PubMed Central PMCID: PMCPMC4753060.

33. Achenbach TM, Edelbrock CS. Manual for the child behavior checklist and revised child behavior profile. Vermont: University of Vermont, Department of Psychiatry; 1983.

34. Su LY, Li XR. Wan GB,Yang ZW,Luo XR.Hunan norm of Achenbach child behavior checklist. Chin J Clinical Psychology. 1996;4(1):24-8. (In Chinese).

35. Xin RE. Tang HQ.Investigate on 24013 city children's behavioral problems in 26 units of 22 provinces.Shanghai Arch. Psychiatr. 1992;4(1):47-55. (In Chinese).

36. Wang XD, Wang XL, Ma H. Handbook of mental health rating scale: revised edition[M]. Beijing: Chin Ment Health J. 1999, 13, (1), 161-167,306-307. (In Chinese). 
37. Gong YX. revised eysenck personality questionnaire handbook [M]. Changsha: hunan medical college.1986, 2. (In Chinese).

38. Su LY, Luo XR, Zhang GS, Xie GR,Liu YZ. Norms of the Piers-Harris Children's Self-concept Scale of Chinese Urban Children. Chin Ment Health J. 2002;16(1):31-4. (In Chinese).

39. Yu X, Dai XY, Li QL, Wang LL,Li LG,Liu MM,Zhang J. Psychological behavior problems and influencing factors in rural Muslim left-behind children. Chin J Public Health. 2014;30(7):857-60. (In Chinese).

40. Collaborative investigation unit of 22 cities in China. Children's behavior problems and their influential factors: A collaborative suvey of 24,013 children and adolescents in 22 cities in China. Chin Ment Health J. 1993;7(1):13-5+46. (In Chinese).

41. Xu J, Chu KK,Xu BZhang JP, Wang CY,Liu QX,et al. Prevalence and related factors of behavioral problems among adolescents aged 12-16 years in Nanjing city. Chin J Public Health. 2018;34(4):489-92. (In Chinese).

42. Guo GW, Yang SW, Liu XY, Wu DX,Wei YL,Yu YS,et al. Epidemiological investigation of behavior problems of aquatic children in rural areas. J Qiannan Medical College for Nationalities. 2012;25(4):284-5..(In Chinese).

43. Wu LJ, Liu AS,Tao YC, Zhang LP,WuK,Chen, Li. An Epidemiological Survey of Behavioral Problems among School-aged Children in Harbin. Chin J School Health.2002,23(6):488-489. (In Chinese).

44. Hu H, Gao J, Jiang H, Jiang H, Guo S, Chen K, et al. A Comparative Study of Behavior Problems among Left-Behind Children, Migrant Children and Local Children. Int J Environ Res Public Health. 2018;15(4):655. doi:10.3390/ijerph15040655. PubMed PMID: 29614783; PubMed Central PMCID: PMCPMC5923697. Epub 2018/04/05.

45. Liu SM, Wang RZ, Wang. YH,et al.An epidemiological investigation of behavioral.

46. problems and the relevant factors in children aged $6 \sim 11$ years in Shandong province. Journal of Psychiatry.2018,31(4):272-275. (In Chinese).

47. Hinshaw SP. On the distinction between attentional deficits/hyperactivity and conduct problems/aggression in child psychopathology. Psychological bulletin. 1987;101(3):443-63. Epub 1987/05/01. PubMed PMID: 3602250.

48. Wang XL, Li PF, ,Peng Y, Li K. Emotional and behavioral problems of children-adolescents aged 4-18 in Changsha. Chin Ment Health J. 2012;26(10):775-9..(In Chinese).

49. Gao Z, Liu ZN,Lin JL, Li H, Li XT. Mei WH.Research and analysis on behavior problems of middle-school students in Zhuhai City and their related factors. Chin J Behavioral Medicine Science. 2004;13(5):93-4. (In Chinese).

50. Zhao C, Wang F, Zhou X, Jiang M, Hesketh T. Impact of parental migration on psychosocial well-being of children left behind: a qualitative study in rural China. Int J Equity Health. 2018;17(1):80.

doi:10.1186/s12939-018-0795-z. PubMed PMID: 29903019; PubMed Central PMCID: PMCPMC6003177. Epub 2018/06/16.

51. Zhou JY, Luo XR,Wei Z,Guan BQ,Yuan XH, Ye HS,et al.Characteristics of Behavioral and Emotiona IProblems of Left-Behind Children in Rural Area of Changsha. J Appl Clin Pediatr.2009,24(24):1901-1903. (In Chinese). 
52. Cabrera N, Hofferth SL, Hancock G. Family structure, maternal employment, and change in children's externalizing problem behaviour: Differences by age and self-regulation. Eur J Dev Psychol. 2014;11(2):136-58. doi: 10.1080/17405629.2013.873716. PubMed PMID: 29170680; PubMed Central PMCID: PMCPMC5696629.

53. Zhao XX, Chen JQ, Feng YN, Jin, YC,Liu. CF,Yu PY.Investigation on behavvioral problems and influencing factors among primary school students in rural areas of shangdong province. Chin J child health care.2015,23(5):522-525. (In Chinese).

54. Zhou YM, Qi YJ, Zhang ZX,He F,Zheng YAnalysis of incidence and influencing factors of psychological behavior problems of left-behind children aged 4-6 in rural areas. Journal of Clinical Psychiatry.2019,29(3):157-160. (In Chinese).

55. Lan YL, Zhang HY, Li P, Xu HB. Self-concept and Behaviour problems of Children. Chin J Clin Psychol. 2004;12(1):53-5. (In Chinese).

56. Zhu WF, Kong LN,Fu YX,Hu XM,Li T, Wang YC,et al. A twin study on externalizing problem behavior in adolescents in Chongqing City. JOURNAL OF HYGIENE RESEARCH. 2017;46(3):423-8. (In Chinese).

57. Gershoff ET. Corporal punishment by parents and associated child behaviors and experiences: a metaanalytic and theoretical review. Psychological bulletin. 2002;128(4):539-79. doi:10.1037/00332909.128.4.539. PubMed PMID: 12081081. Epub 2002/06/26.

58. Callender KA, Olson SL, Choe DE, Sameroff AJ. The effects of parental depressive symptoms, appraisals, and physical punishment on later child externalizing behavior. J Abnorm Child Psychol. 2012;40(3):47183. doi:10.1007/s10802-011-9572-9. PubMed PMID: 21947616; PubMed Central PMCID:

PMCPMC4100716. Epub 2011/09/29.

59. Ji FT. The fundamental characteristics of Hui culture. Northwest Ethnic Studies. 2018;(01):96-103.

60. Li JL, Ma CQ. Family Education and the Formation of Hui Culture concept. Journal of Northwestern University (Philosophy and Social Sciences Edtion). 1997;(03):118-21.

61. Mingebach T, Kamp-Becker I, Christiansen H, Weber L. Meta-meta-analysis on the effectiveness of parentbased interventions for the treatment of child externalizing behavior problems. PLoS One. 2018;13(9):e0202855. doi:10.1371/journal.pone.0202855. PubMed PMID: 30256794; PubMed Central PMCID: PMCPMC6157840. Epub 2018/09/27.

62. Grusec JE. Socialization processes in the family: social and emotional development. Annual review of psychology. 2011;62:243 - 69. Epub 2010/08/25. doi: 10.1146/annurev.psych.121208.131650. PubMed PMID: 20731599.

63. Shek DTL, Zhu X. Reciprocal Relationships Between Moral Competence and Externalizing Behavior in Junior Secondary Students: A Longitudinal Study in Hong Kong. Front Psychol. 2019;10:528. doi:10.3389/fpsyg.2019.00528. PubMed PMID: 30894835; PubMed Central PMCID: PMCPMC6414454. Epub 2019/03/22.

\section{Supplementary Files}

This is a list of supplementary files associated with this preprint. Click to download. 
- EBPofHuiLBcSupplementarymaterials.rar

- Thequestionnaire.doc 